\title{
Magnon-assisted Andreev reflection in a ferromagnet-superconductor junction
}

\author{
Edward McCann and Vladimir I. Fal'ko \\ Department of Physics, Lancaster University, Lancaster, LA1 4YB, United Kingdom
}

(October 29, 2018)

\begin{abstract}
We study subgap transport in a ferromagnet-superconductor junction at low temperature due to Andreev reflection. The mismatch of spin polarized current in the ferromagnet and spinless current in the superconductor results in an additional contact resistance which can be reduced by magnon emission in the ferromagnet. Using the s-f model and focusing on half-metallic ferromagnets, we calculate the corresponding nonlinear contribution to the current which is asymmetric with respect to the sign of the bias voltage and is related to the density of states of magnons in the ferromagnet.
\end{abstract}

PACS numbers: 73.23.-b, 74.80.Fp, 73.63.Rt, 72.25.-b.

Spin polarized transport is a subject of intense research, motivated largely by the possibility of developing a form of electronics which utilizes the spin polarization of carriers]. Ferromagnetic (F) metals have more carriers of one spin polarization (known as majority carriers) present at the Fermi energy $E_{F}$ than of the inverse polarization (minority carriers). Of particular interest are so called half-metals where the splitting $\Gamma$ between the majority and minority conduction bands is greater than $E_{F}$ measured from the bottom of the majority band. In such a material only majority carriers are present at the Fermi energy and electric current in it is completely spin polarized. An F-F junction with antiparallel spin polarizations has a larger contact resistance than a junction with parallel polarizations due to a mismatch of spin currents at the interface, leagding to tunneling magnetoresistance in F-F junction ${ }^{2} 3$ and giant magnetoresistance in multilayer structures 1 . Spin current mismatch may also affect the fonductance of a ferromagnet-superconductor junction 6 . At low temperatures and small bias voltage, current flows through the interface due to Andreev reflection 10 whereby particles in the ferromagnetic region with excitation energies $\epsilon$ smaller than the superconducting gap energy $\Delta$ are reflected from the interface as holes. Since subgap transport in the superconductor (S) is mediated by spinless Cooper pairs the spin current is zero in the superconductor in coptrast to the ferromagnet.

Spin relaxation processes 111 , such as spin-orbit scattering at impurities or magnon emission, can reduce the spin current mismatch. In a given junction, spin-orbit scattering (which is an elastic process) wowld reduce the value of the additional contact resistance 12 whereas the inelastic process of magnon emission would manifest itself as a modification of the form of the $I(V)$ characteristics. Nonlinear $I(V)$ characteristics due to magnon-assisted tunneling between two ferromagnets have already been studied both theoretically 13 and experimentally 14 with a view to relate the second derivative of the current to the density of states of magnons $\Omega(\omega)$ as $d^{2} I / d V^{2} \propto \Omega(e V)$. In the present work we analyze the role of magnon emission in the formation of the subgap $I(V)$ characteristics, $\mathrm{eV}<\Delta$, of a ferromagnet-superconductor junction with emphasis given to half-metallic ferromagnets. Microscop- ically, the relevant process involves the transfer of a singlet pair of electrons from the superconductor to the ferromagnet where one of them forms an intermediate state at an energy below the bottom of the conduction band for minority electrons. Then, this latter electron can emit a magnon in the ferromagnet which will carry away excess spin allowing the electron to incorporate ifself into the majority conduction band. The s-f model 15,16 is employed to describe a ferromagnet assuming that electrical conduction and magnetism are caused by different groups of electrons which interact via s-f exchange.

To anticipate a little, due to magnon-assisted Andreev reflection at zero temperature, the $I(V)$ characteristics of an F-S junction for $e V<\Delta$ acquire an additional contribution which is specified by the differential of the nonlinear differential conductance,

$$
\begin{aligned}
\frac{d^{2} I}{d V^{2}}= & \frac{e v G_{A}}{S}\left\{\frac{\Omega(2 e V)}{\left[1+\frac{\left(2 e V-\omega_{0}\right) W}{\Gamma \omega_{m}}\right]^{2}\left[1-\left(\frac{e V}{\Delta}\right)^{2}\right]}+\right. \\
& \left.+\int_{0}^{2 e V} \frac{\Omega(\epsilon)\left[F\left(\frac{e V}{\Delta}\right)-F\left(\frac{\epsilon-e V}{\Delta}\right)\right] \frac{d \epsilon}{2 \Delta}}{\left[1+\frac{\left(\epsilon-\omega_{0}\right) W}{\Gamma \omega_{m}}\right]^{2}}\right\} .
\end{aligned}
$$

Here $F(x)=x /\left(1-x^{2}\right)^{2}, \omega_{m}$ is the magnon bandwidth and $\omega_{0}$ is the anisotropy energy responsible for a gap in the magnon density of states $[(\omega)$ is non-zero for $\omega_{0}<\omega<\omega_{m}$ and zero otherwise it. The factor of two in $\Omega(2 \mathrm{eV})$ arises because an elementary Andreev reflection process involves a net transfer of charge $2 e$ across the biased junction. The conductance $G_{A}$ incorporates tunneling parameters relevant for the Andreev process and it is defined explicitly later. $W$ is the conduction band width of electrons, $v$ is the volume of a unit cell, and $\Gamma=2 \mathcal{J} S$ is the conduction electron band splitting where $S$ is the spin of the local moments, and $\mathcal{J}$ is the s-f exchange constant.

The nonlinear contribution to the current, Eq. (11), is asymmetric with respect to bias voltage $V$. It is zero for negative bias and finite for $V>\omega_{0} /(2 e)$. This feature can be expained using the sketch in Fig 1 which illustrates the tunneling process between an $\mathrm{S}$ electrode on 
the left hand side and an F electrode on the right for (a) $V>0$ and (b) $V<0$. We have adopted the convention that positive (negative) voltage results in a Fermi energy $E_{F}$ in the ferromagnet that is lower (higher) by energy $|e V|$ than the Fermi energy $E_{S}$ in the superconductor. For $V>0$, Fig 1(a), Andreev reflection results in the injection of both a majority (spin 'up') and a minority (spin 'down') electron into the ferromagnet. One of these electrons has an energy $\epsilon$ above the Fermi energy in the superconductor $E_{S}$, the other has an energy $\epsilon$ below $E_{S}$. With respect to the Fermi energy in the ferromagnet $E_{F}$ these energies are $e V+\epsilon$ and $e V-\epsilon$, respectively, which are both above $E_{F}$ because of the need to move into unoccupied states in the ferromagnet, $\epsilon \leq \mathrm{eV}$. At zero temperature $T=0$ the core spins are all aligned in the majority, up direction and, if $E_{F} \ll \Gamma$, then only spin up conduction electrons are present at $E_{F}$ in the ferromagnet. A dynamic process which allows a spin down electron to enter the ferromagnet is shown schematically in Fig 1(a). The spin down electron tunnels from the superconductor into a virtual, intermediate spin down state above $E_{F}$, then it emits a magnon and incorporates itself into an empty state in the majority conduction band. In Fig 1(a) the magnon is depicted as a flip in the spin of one of the localized magnetic moments.

On the other hand, for $V<0$, Fig 1(b), Andreev reflection would result in the injection of a spin up and a spin down electron from the ferromagnet into the superconductor with energies $\epsilon$ above and below $E_{S}$. With respect to the Fermi energy in the ferromagnet $E_{F}$ these energies are $-|e V|+\epsilon$ and $-|e V|-\epsilon$, respectively, which are both below $E_{F}$ because of the need to have initially occupied states in the ferromagnet, $\epsilon \leq|e V|$. As stated above, there are no spin down states at these energies near $E_{F}$. Since a spin up electron cannot emit a magnon (left side of Fig 1(b)), due to conservation of total spin in the exchange interaction, the only possibility would be that a spin up electron in the ferromagnet would absorb a magnon to form an intermediate spin down state before tunneling into the superconductor. However there are no thermally excited magnons at $T=0$ in the initial state of the ferromagnet (right side of Fig 1(b)) so it is impossible for magnon-assisted Andreev reflection to contribute to current formation in negatively biased junctions.

To calculate the current across an F-S junction, we use the transfer Hamilonian method and the nonequilibrium Keldysh technique 18 [19 to express the current in terms of the tunneling density of states in the metallic ferromagnet which will be determined using the s-f model. We will generalize the approach of Ref. 19 developed for normalsuperconductor junctions to the case of an F-S junction. We consider a point contact geometry which consists of a narrow constriction between $\mathrm{S}$ and $\mathrm{F}$ electrodes and we use the total Hamiltonian of the system in the form of

$$
\begin{aligned}
H & =H_{S}+H_{F}+H_{T}, \\
H_{T} & =\sum_{\alpha}\left(t c_{S, \alpha}^{\dagger} c_{F, \alpha}+t^{*} c_{F, \alpha}^{\dagger} c_{S, \alpha}\right),
\end{aligned}
$$

in all calculations paying attention to the spin index $\alpha=\{\uparrow, \downarrow\}$. In the tunneling Hamiltonian $H_{T}, t$ describes the transfer of an electron from $\mathrm{S}$ to $\mathrm{F}$. We assume that $t$ is independent of energy and that spin is conserved upon electron transfer across the interface.

Assuming that the voltage drop $V$ occurs across the point contact and both electrodes are almost in equilibrium, for a given distribution and density of states in the reservoirs, the current can be calculated as the mean value of the current operator, $I=$ $(i e / \hbar) \sum_{\alpha}\left(t\left\langle c_{S, \alpha}^{\dagger} c_{F, \alpha}\right\rangle-t^{*}\left\langle c_{F, \alpha}^{\dagger} c_{S, \alpha}\right\rangle\right)$. The averages in this equation may be expressed in terms of nonequilibrium Keldysh Greens functions $\hat{G}^{+-}$and it is convenient for the case of a superconducting electrode to use the $2 \times 2$ Nambu representation 19 . After some algebra we arrive at the following expression for the current

$$
\begin{aligned}
I(V)= & \frac{e}{h} 4 \pi^{2}|t|^{4} \int_{-\infty}^{\infty} d \epsilon\left|\hat{G}_{S, 12}^{R}(\epsilon)\right|^{2} \\
& \times[f(\epsilon-e V)-f(\epsilon+e V)] \\
& \times\left[\rho_{\uparrow}\left(E_{F}+\epsilon+e V\right) \rho_{\downarrow}\left(E_{F}-\epsilon+e V\right)+\right. \\
& \left.\quad+\rho_{\downarrow}\left(E_{F}+\epsilon+e V\right) \rho_{\uparrow}\left(E_{F}-\epsilon+e V\right)\right] .
\end{aligned}
$$

This expression describes the current due to Andreev reflection of electrons at the F-S interface. The gapfull spectrum of quasi-particles in the superconductor is taken into account in the off-diagonal element of the retarded Greens function $\hat{G}_{S, 12}^{R}$ and the energy $\epsilon$ is measured with respect to the Fermi energy $E_{S}$ in the superconductor.

In Eq. (2), $\rho_{\alpha}(E)$ is the tunneling density of states of spin state $\alpha$ in the ferromagnet. For $\Gamma \gg\left\{E_{F}, e V\right\}$ and in the absence of spin waves, $\rho_{\downarrow}(E)$ is zero in a half metal at energies close to the chemical potentials of the electrodes and $I(V)=0$. However, as explained above, $\rho_{\downarrow}(E)$ is nonzero for $E>E_{F}+\omega_{0}$ because of the dynamical process of magnon-assisted tunneling. The tunneling density of states $\rho_{\downarrow}(E)$ is determined using the s-f mode 15,16 with the following Hamiltonian

$$
\begin{aligned}
H_{F} & =H_{0}+H_{J}+H_{e x}, \\
H_{0} & =\sum_{\langle i j\rangle \alpha} t_{i j} c_{i \alpha}^{\dagger} c_{j \alpha} \equiv \sum_{k \alpha} \epsilon_{k} c_{k \alpha}^{\dagger} c_{k \alpha}, \\
H_{J} & =-J \sum_{\langle i j\rangle} \mathbf{S}_{\mathbf{i}} \cdot \mathbf{S}_{\mathbf{j}}-\omega_{0} \sum_{i} S_{i}^{z}, \\
H_{e x} & =-\mathcal{J} \sum_{j \alpha \beta}\left(\sigma_{\alpha \beta} \cdot \mathbf{S}_{\mathbf{j}}\right) c_{j \alpha}^{\dagger} c_{j, \beta},
\end{aligned}
$$

where $H_{0}$ deals with conduction band electrons, $H_{J}$ is the Heisenberg Hamiltonian of the localized moments and $H_{e x}$ takes into account the s-f intra-atomic exchange. We will consider weak, ferromagnetic s-f coupling $0<\mathcal{J} S<$ $W$, thus excluding the case corresponding to double exchange $\mathcal{J} S \gg W$.

The conduction electron single particle Greens function and energy spectrum have been studied in the 
limit $\rho$ zero temperature both for zero conduction band filling 15 and finite filling 16 . In the regime $\left\{e V, \omega_{0}\right\} \ll$ $E_{F} \ll \Gamma \ll W$ the minority electron density of states is

$$
\rho_{\downarrow}(E) \approx \frac{v \Gamma^{2}}{2 S L^{d}} \sum_{k^{\prime}} \sum_{q} \frac{\left[1-f\left(k^{\prime}\right)\right] \delta\left(\epsilon_{k^{\prime}}-E+\omega_{q}\right)}{\left(\epsilon_{k^{\prime}+q}-E+\Gamma\right)^{2}}
$$

where the energy $E$ is measured from the bottom of the spin up conduction band and $L^{d}$ is the volume of the system. A similar expression, with $2 S=1$, was also obtained for itinerant ferromagnets using the Hubbard model20. The energy in the denominator of Eq. (3), $\epsilon_{k^{\prime}+q}-E+\Gamma$, is related to the inverse lifetime of an electron in the virtual state, the delta function in the numerator accounts for energy conservation of the entire process and the factor $\left[1-f\left(k^{\prime}\right)\right]$ indicates that this process is a dissipative one and only involves unoccupied final (spin up) states. Therefore the minority density of states is finite for energies $E>E_{F}+\omega_{0}$ only. At $T=0$, in our regime of interest, spin waves do not affect the majority density of states $\rho_{\uparrow}(E) \approx N(E)$, where $N(E)$ is the bare electron density of states arising from the Hamiltonian $H_{0}$. We have neglected interactions between conduction electrons so that the dominant effect of finite filling is the requirement to obey Fermi statistics.

The dispersion of magnons in a ferromagnet is quadratic at low frequencies, $\omega_{q}=D q^{2}+\omega_{0}$ where $D=2 J S^{2} a^{2}$ and $a$ is the lattice constant. For simplicity, we shall also use the Debye approximation for a broader range of energies of the magnon spectrum by introducing a cutoff of the parabolic magnon dispersion at $\omega_{m} \approx D\left(6 \pi^{2} / v\right)^{2 / 3}$ since, for $\Delta \ll \omega_{m}$, such a simplification does not influence the final results. We also use a parabolic approximation for free electrons in a conduction band and find that, for a three dimensional ferromagnetic metal and $E \approx E_{F}$,

$$
\begin{aligned}
\rho_{\downarrow}(E) \approx \frac{v N(E)}{2 S} \int_{0}^{E-E_{F}} & \frac{\Omega(\omega) d \omega}{\left[1+\left(\frac{W}{\Gamma}\right) \frac{\omega-\omega_{0}}{\omega_{m}}\right]^{2}}, \\
\Omega(\omega) & =\frac{\sqrt{\omega-\omega_{0}}}{4 \pi^{2} D^{3 / 2}} \quad ; \quad \omega_{0}<\omega<\omega_{m} .
\end{aligned}
$$

We use Eq. (2) to calculate the current due to Andreev reflection for low bias voltage $e V<\Delta$, taking into account the lowest order in the tunneling matrix elements, $|t|^{4}$. In a BCS superconductor the off-diagonal Greens function is $\hat{G}_{S, 12}^{R}(\epsilon) \approx \pi N\left(E_{S}\right) / \sqrt{1-(\epsilon / \Delta)^{2}}$ for $\epsilon<\Delta$, where $N\left(E_{S}\right)$ is the density of states in the normal state near the Fermi energy. Note that if the ferromagnet were to be replaced by a normal metal where the density of states at $E_{F}$ is $N\left(E_{F}\right)$ for both spin channels, the current at low bias would be $I=V G_{A}$ with conductance $G_{A} \approx\left(e^{2} / h\right) 16 \pi^{4}|t|^{4} N^{2}\left(E_{F}\right) N^{2}\left(E_{S}\right)$. As we assume $e V \ll E_{F}, \rho_{\uparrow} \approx N\left(E_{F}\right)$, and Eq. (2) for an F-S junction can be simplified, by shifting the energy variable, into

$$
I(V) \approx \frac{G_{A}}{2 e N\left(E_{F}\right)} \int_{0}^{2 e V} \frac{d \epsilon \rho_{\downarrow}\left(E_{F}+\epsilon\right)}{\left[1-\left(\frac{e V-\epsilon}{\Delta}\right)^{2}\right]} .
$$

Using $\rho_{\downarrow}\left(E_{F}+\epsilon\right)$ from Eq. (4), we find for $e V<\Delta \ll$ $\omega_{m} \Gamma / W$ that 17

$$
\begin{aligned}
I(V) & \approx \frac{v \Delta G_{A}}{24 e \pi^{2} S D^{3 / 2}} \theta\left(2 e V-\omega_{0}\right)\left\{-2 \Delta \sqrt{2 e V-\omega_{0}}+\right. \\
& +\left(\Delta-e V+\omega_{0}\right)^{3 / 2} \arctan \left(\sqrt{\frac{2 e V-\omega_{0}}{\Delta-e V+\omega_{0}}}\right) \\
& \left.+\left(\Delta+e V-\omega_{0}\right)^{3 / 2} \operatorname{arctanh}\left(\sqrt{\frac{2 e V-\omega_{0}}{\Delta+e V-\omega_{0}}}\right)\right\} .
\end{aligned}
$$

The $I(V)$ characteristics obtained yield the relation between the magnon density of states and $d^{2} I / d V^{2}$ shown in Eq. (11). For small voltages $e V \ll \Delta$, the current may be simplified further as

$$
I(V) \approx \frac{v G_{A}}{60 e \pi^{2} S D^{3 / 2}}\left(2 e V-\omega_{0}\right)^{5 / 2} \theta\left(2 e V-\omega_{0}\right),
$$

and $d^{2} I / d V^{2} \propto \Omega(2 e V)$. The factor $\theta\left(2 e V-\omega_{0}\right)$ emphasises the absence of a contribution to the current from magnon-assisted Andreev reflection for negative bias. At zero temperature, the $I(V)$ characteristics are asymmetric, which can be used to extract magnon-assisted Andreev reflection in an F-S junction involving a ferromagnet with electrons of both polarizations present at $E_{F}$. Note that at $T>\omega_{0}$, the absorption of thermal magnons would allow for a similar contribution to the current at $V<0$. A graphic representation of the result of Eq. (11) is given in Fig 2, in comparison with a similar analysis for an F-F junction in an antiparallel configuration (inset). In particular, we illustrate how the relation between the conduction electron bandwidth and the exchange splitting energy, $W / \Gamma$, may affect the result, since for $W \gg \Gamma$ the contribution of large energy magnons to the lifting of spin current mismatch is not so efficient as for $W \ll \Gamma$.

To summarise, spin current mismatch in an F-S junction at subgap voltages may be lifted by magnon-assisted tunneling resulting in nonlinear $I(V)$ characteristics related to the magnon density of states. Such features are typical not only for junctions between ferromagnets and BCS superconductors, but also for HiTc materials, since subgap transport through any spin singlet superconductor would be affected by spin current mismatch at the F-S interface. For a matching pair of materials, with the Curie temperature of the ferromagnet similar to the transition temperature of the HiTc material, the Andreev reflection process could be used to probe the whole magnon spectrum.

The authors thank N. R. Cooper, D. M. Edwards, C. J. Lambert, and Yu. V. Nazarov for discussions. This work was supported by EPSRC, with partial travel support from NATO and COST. 
${ }^{1}$ G. A. Prinz, Physics Today, April, 58 (1995). Science 282, 1660 (1998), and Refs. therein.

${ }^{2}$ M. Julliere, Phys. Lett. 54A, 225 (1975).

${ }^{3}$ J. S. Moodera, T. H. Kim, C. Tanaka, and C. H. de Groot, Philos. Mag. B 80, 195 (2000).

${ }^{4}$ M. N. Baibich, J. M. Broto, A. Fert, N. Van Dau, F. Petroff, P. Eitenne, G. Creuzet, A. Friederich, and J. Chazelas, Phys. Rev. Lett. 61, 2472 (1988). G. Binasch, P. Grünberg, F. Saurenbach, and W. Zinn, Phys. Rev. B 39, 4828 (1989).

${ }^{5}$ W. P. Pratt Jr., S. F. Lee, J. M. Slaughter, R. Loloee, P. A. Schroeder, and J. Bass, Phys. Rev. Lett. 66, 3060 (1991). S. F. Lee, W. P. Pratt Jr., R. Loloee, P. A. Schroeder, and J. Bass, Phys. Rev. B 46, 548 (1992).

${ }^{6}$ M. J. M. de Jong and C. W. J. Beenakker, Phys. Rev. Lett. 74, 1657 (1995).

${ }^{7}$ V. I. Fal'ko, A. F. Volkov, and C. J. Lambert, Phys. Rev. B 60, 15394 (1999). E. McCann, V. I. Fal'ko, A. F. Volkov, and C. J. Lambert, Phys. Rev. B 62, 6015 (2000).

${ }^{8}$ F. J. Jedema, B. J. van Wees, B. H. Hoving, A. T. Filip, and T. M. Klapwijk, Phys. Rev. B 60, 16549 (1999).

${ }^{9}$ V. I. Fal'ko, C. J. Lambert, and A. F. Volkov, Pis'ma v ZhETF 69, 497 (1999) [JETP Letters 69, 532 (1999)].

${ }^{10}$ A. F. Andreev, Zh. Exp. Teor. Fiz. 46, 1823 (1964) [Sov. Phys. JETP 19, 1228 (1964)].

${ }^{11}$ M. Johnson and R. H. Silsbee, Phys. Rev. Lett. 55, 1790 (1985).

12 D. V. Baxter, S. D. Steenwyk, J. Bass, and W. P. Pratt Jr., J. Appl. Phys. 85, 4545 (1999).

13 A. M. Bratkovsky, Appl. Phys. Letts. 72, 2334 (1998). F. Guinea, cond-mat/9712075.

${ }^{14}$ D. C. Tsui, R. E. Dietz, and L. R. Walker, Phys. Rev. Lett. 27, 1729 (1971). J. S. Moodera, J. Nowak, and R. J. M. van de Veerdonk, Phys. Rev. Lett. 80, 2941 (1998).

15 B. S. Shastry and D. C. Mattis, Phys. Rev. B 24, 5340 (1981). S. R. Allan and D. M. Edwards, J. Phys. C: Solid State Phys. 15, 2151 (1982).

${ }^{16}$ R. B. Woolsey and R. M. White, Phys. Rev. B 1, 4474 (1970). M. I. Auslender and V. Y. Irkhin, J. Phys. C: Solid State Phys. 18, 3533 (1985).

17 Strictly speaking, the condition $e V<\Delta$ should be replaced by $e V<\Delta \sqrt{1-\pi^{2}|t|^{2} N^{2}\left(E_{F}\right)}$ where we consider $\pi^{2}|t|^{2} N^{2}\left(E_{F}\right) \ll 1$.

${ }^{18}$ C. Caroli, R. Combescot, P. Nozieres, and D. Saint-James, J. Phys. C 4, 916 (1971).

19 J. C. Cuevas, A. Martín-Rodero, and A. Levy Yeyati, Phys. Rev. B 54, 7366 (1996).

${ }^{20}$ D. M. Edwards and J. A. Hertz, J. Phys. F: Metal Phys. 3, 2191 (1973).

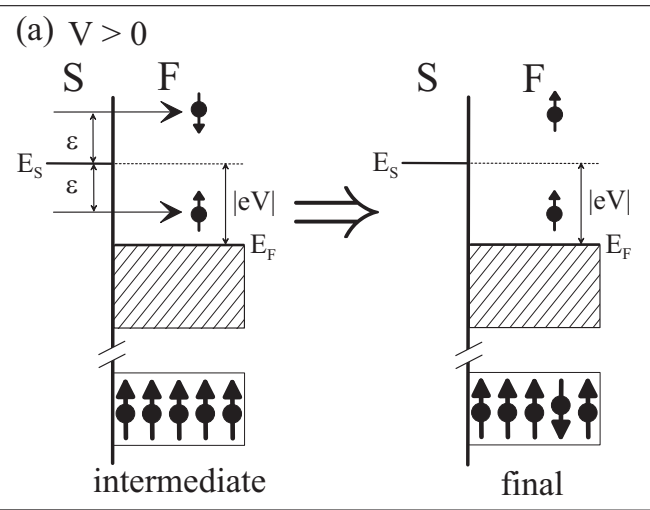

(b) $\mathrm{V}<0$

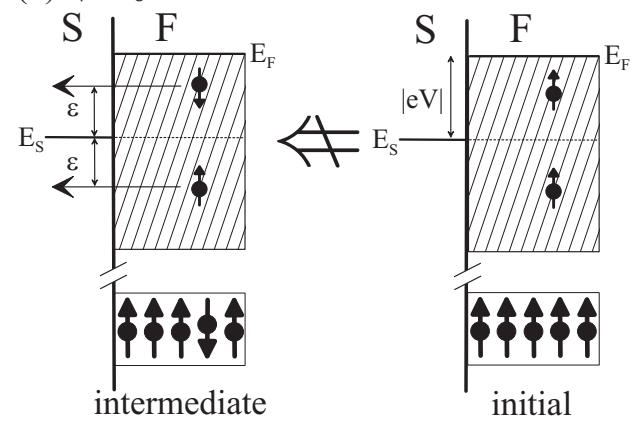

FIG. 1. Schematic of the tunneling process between a superconducting electrode on the left hand side and a ferromagnetic electrode on the right for (a) $V>0$ and (b) $V<0$. For (a) $V>0$ a down spin electron tunneling into the ferromagnet may emit a magnon and incorporate itself into the majority conduction band. For (b) $V<0$ no spin flip process is possible at $T=0$ because in the initial state (right) there are no thermal magnons for an up spin electron to absorb. See text for details.

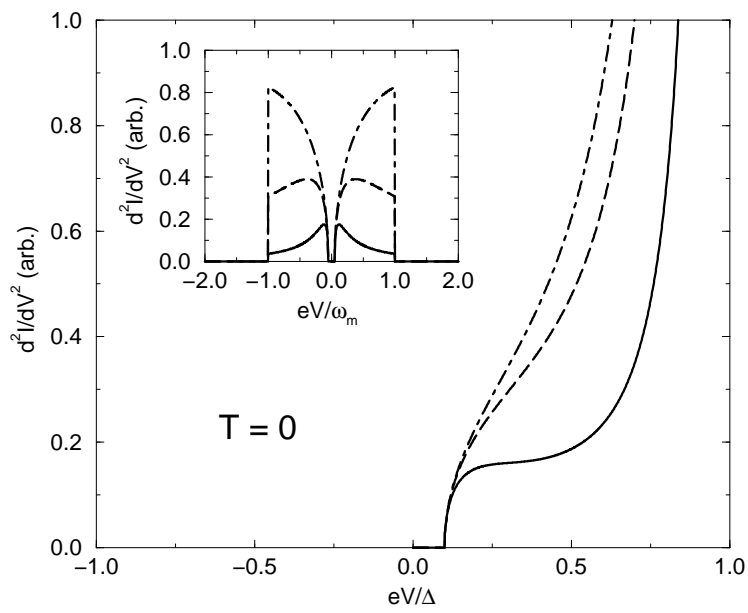

FIG. 2. Typical form of $d^{2} I / d V^{2}$ for an F-S junction with various choices of material parameters. From top to bottom, $\Gamma / W=5.0$ (dot-dashed), 1.0 (dashed), and 0.2 (solid). As an example, we choose $\omega_{0}=0.2 \Delta$ and $\omega_{m}=4.0 \Delta$. For comparison, inset shows $d^{2} I / d V^{2}$ for an F-F junction with antiparallel spin polarizations. 